\title{
El Órgano administrativo de Recursos Contractuales de Cataluña: un nuevo avance en la garantía del derecho a una buena administración
}

\author{
Dr. Juli Ponce Solé \\ Profesor Titular de Derecho Administrativo. \\ Acreditado para Catedrático \\ Universidad de Barcelona \\ e-mail:jponce@ub.edu
}

\author{
Oscar Capdeferro Villagrasa \\ Becario FPU (Ministerio de Educación) \\ Derecho Administrativo \\ Universidad de Barcelona \\ e-mail: ocapdeferro@ub.es
}

\begin{abstract}
Resumen Entre las reformas introducidas por la Ley estatal 34/2010 en el sistema de contratación pública figura la creación del Tribunal Administrativo Central de Recursos Contractuales, así como el mandato a las comunidades autónomas para el establecimiento de órganos análogos en distintos ámbitos dentro de su territorio. En su cumplimiento, la Comunidad Autónoma de Cataluña ha creado, mediante sendas leyes, el Tribunal de Recursos Contractuales del Parlamento de Cataluña y el Órgano Administrativo de Recursos Contractuales de Cataluña. Se trata de órganos especializados en materia de revisión de procedimientos de contratación, configurados con plena independencia funcional en el ejercicio de sus funciones, que son, principalmente, la resolución de los recursos especiales en materia de contratación y las cuestiones de nulidad correspondientes. La configuración de órganos administrativos independientes como esos supone un avance en el reforzamiento y protección del derecho a una buena administración.
\end{abstract}

\section{The Public Procurement Review Administrative Authority of Catalonia: another step towards guaranteeing the right to a Good Public Administration}

\begin{abstract}
The reforms introduced in the public procurement system under State Law 34/2010 include the creation of the Public Procurement Review Central Administrative Court, and the requirement imposed on the autonomous regions to establish similar authorities for different areas in their territory. In compliance with this requirement, the Autonomous Region of Catalonia has created, under the respective laws, the Public Procurement Review Court of the Parliament of Catalonia and the Public Procurement Review Administrative Authority of Catalonia. These bodies are specialised in reviewing public procurement procedures and are set up to carry out their functions with full independence. Their main functions are the settlement of special public procurement reviews and the respective issues of nullity. The creation of these independent administrative authorities is another step towards strengthening and protecting the right to a good public administration.
\end{abstract}

Key words

Independent administrative authorities - public procurement-administrative reviews. 


\section{INTRODUCCIÓN}

Como es sabido, la Ley 30/2007, de 30 de octubre, de Contratos del Sector Público, derogó, en gran parte, el Texto Refundido de la Ley de Contratos de las Administraciones Públicas, que había sido aprobado por el Real Decreto Legislativo 2/2000, de 16 de junio'. Como declaraba sin ambages la exposición de motivos de la Ley de 2007, es conocido que el motivo principal de la reforma fue la adaptación al Derecho comunitario $^{2}$. La Ley 30/2007, en los cuatro años que estuvo en vigor, sufrió numerosas reformas $^{3}$. No obstante, la que interesa a efectos de este estudio, fue la operada por la Ley 34/2010, de 5 de agosto, de modificación de las Leyes 30/2007, de 30 de octubre, de Contratos del Sector Público, 31/2007, de 30 de octubre, sobre procedimientos de contratación en los sectores del agua, la energía, los transportes y los servicios postales, y 29/1998, de 13 de julio, reguladora de la Jurisdicción Contencioso-Administrativa para adaptación a la normativa comunitaria de las dos primeras.

Como claramente indica el mismo nombre de la Ley, su finalidad principal fue la adaptación de la legislación de contratos española al Derecho comunitario en la materia, singularmente a la Directiva 2007/66/CE, del Parlamento Europeo y del Consejo, de 11 de diciembre de 2007, de modificación de las Directivas 89/665/CEE y 92/13/CEE del Consejo en lo que respecta a la mejora de la eficacia de los procedimientos de recurso en materia de adjudicación de contratos públicos.

Como es sabido, la Ley 34/2010 introdujo la cuestión de nulidad y renovó el recurso especial en materia de contratación; además, atribuyó su resolución a un órgano administrativo independiente. Esta misma ley se encargó de crear dicho órgano independiente para el ámbito de la Administración General del Estado (el Tribunal Admi-

1 Excepto el Cap. IV del Título V del Libro II, arts. 253 a 260 (v. DD Única Ley 30/2007).

2 “[...] siendo la necesidad de incorporar a nuestro ordenamiento la Directiva 2004/18/CE el motivo determinante de la apertura de un nuevo proceso de revisión de nuestra legislación de contratos públi$\cos [\ldots] "$.

3 Fue modificada por: Ley 14/2010, de 5 de julio, sobre las infraestructuras y los servicios de información geográfica en España; Ley 15/2010, de 5 de julio, de modificación de la Ley 3/2004, de 29 de diciembre, por la que se establecen medidas de lucha contra la morosidad en las operaciones comerciales; Ley 34/2010, de 5 de agosto, de modificación de las Leyes 30/2007, 31/2007 y 29/1998; Ley 2/2011, de 4 de marzo, de Economía Sostenible; Ley 26/2011, de 1 de agosto, de adaptación normativa a la Convención Internacional sobre los Derechos de las Personas con Discapacidad; Real Decreto 817/2009, de 8 de mayo, por el que se desarrolla parcialmente la Ley 30/2007, de 30 de octubre, de Contratos del Sector Público; Real Decreto-ley 6/2010, de 9 de abril, de medidas para el impulso de la recuperación económica y el empleo; Real Decreto-ley 8/2010, de 20 de mayo, por el que se adoptan medidas extraordinarias para la reducción del déficit público; Real Decreto-ley 5/2011, de 29 de abril, de medidas para la regularización y control del empleo sumergido y fomento de la rehabilitación de viviendas; y, finalmente, las órdenes $\mathrm{EHA} / 3875 / 2007$, de 27 de diciembre, por la que se hacen públicos los límites de los distintos tipos de contratos a efectos de la contratación administrativa a partir del 1 de enero de 2008 y EHA/3497/2009, de 23 de diciembre, por la que se hacen públicos los límites de los distintos tipos de contratos a efectos de la contratación administrativa a partir del 1 de enero de 2010. Finalmente, fue derogada mediante la DD única del Real Decreto Legislativo 3/2011, de 14 de noviembre, por el que se aprueba el texto refundi194 reformas operadas. 
nistrativo Central de Recursos Contractuales, actualmente en el art. 41.1 RDL 3/2011) y remitía a la normativa autonómica para la creación de este tipo de órganos en los demás ámbitos.

El presente artículo va a analizar de modo sucinto cómo la Comunidad Autónoma catalana ha desarrollado esta ley estatal, estableciendo el Tribunal de Recursos Contractuales en el ámbito del Parlamento de Cataluña (apartado II) y el Órgano de Recursos Contractuales (apartado III).

\section{EL TRIBUNAL DE RECURSOS CONTRACTUALES DEL PARLAMENTO DE CATALUÑA}

El art. 41.3 RDL 3/2011 hace referencia a los ámbitos donde las comunidades autónomas pueden crear los respectivos órganos independientes para la resolución de los recursos especiales en materia de contratación pública4. Por el momento en Cataluña se han creado dos: el del Parlamento y el de la Administración de la Generalidad (y de la Administración local). Si bien es en el segundo en el que se centra este estudio, también es pertinente hacer una breve exposición del órgano del Parlamento.

El Tribunal de Recursos Contractuales del Parlamento de Cataluña fue creado por Acuerdo del Parlamento de Cataluña de 1 de marzo de 20115. Esta breve norma se compone de cuatro artículos y dos disposiciones finales, y configura un órgano colegiado formado por tres miembros del Parlamento de Cataluña: un diputado, un interventor y un letrado, nombrados por la Mesa del Parlamento al inicio de cada legislatura, y cuyo mandato comprende toda la legislatura. De acuerdo con la norma de creación, es un órgano "especializado en materia de revisión de procedimientos de contratación, que actúa con plena independencia funcional en el ejercicio de sus funciones”.

Este órgano es competente para conocer y resolver: los recursos especiales en materia de contratación referidos en el art. 40 RDL 3/2011, las cuestiones de nulidad contractual establecidas en los supuestos especiales del art. 37 RDL 3/2011 y las decisiones pertinentes referidas a las medidas cautelares o provisionales que, en los casos de su competencia, soliciten las personas legitimadas.

4 “En el ámbito de las Comunidades Autónomas, así como en el de los órganos competentes de sus Asambleas Legislativas y de las instituciones autonómicas análogas al Tribunal de Cuentas y al Defensor del Pueblo la competencia para resolver los recursos será establecida por sus normas respectivas, debiendo crear un órgano independiente cuyo titular, o en el caso de que fuera colegiado al menos su Presidente, ostente cualificaciones jurídicas y profesionales que garanticen un adecuado conocimiento de las materias de que deba conocer. El nombramiento de los miembros de esta instancia independiente y la terminación de su mandato estarán sujetos en lo relativo a la autoridad responsable de su nombramiento, la duración de su mandato y su revocabilidad a condiciones que garanticen su independencia e inamovilidad".

5 Publicado en el DOGC núm. 5846, de 28 de marzo de 2011, pp. 17899-17901. 
En cuanto al procedimiento, el Acuerdo se remite a la regulación básica y a las normas de desarrollo en materia de contratos del sector público, y a la legislación en materia de contratación aplicable a la Generalidad de Cataluña. La única especificación propia del Parlamento, se encuentra en el art. 4.2 del Acuerdo, donde se establece el lugar en que se debe presentar el anuncio previo a la interposición del recurso: el Registro General del Parlamento. Para las demás especialidades que sean necesarias, se dota al propio Tribunal con capacidad para adoptarlas por sí mismo, una vez puesto en funcionamiento (art. 4.1).

Sus resoluciones, que deben estar motivadas y fundamentadas en derecho, agotan la vía administrativa.

\section{EL ÓRGANO ADMINISTRATIVO DE RECURSOS CONTRACTUALES DE CATALUÑA}

\section{III.1. La ley reguladora del órgano administrativo de recursos contractuales de Cataluña}

El art. 41 RDL 3/2011 establece para las Comunidades Autónomas la obligación de crear un órgano independiente para la resolución de este tipo de recursos ${ }^{6}$. No obstante la rotundidad con que el primer párrafo del artículo obliga a la creación del órgano, a continuación se establece, en el cuarto párrafo del art. 41.3 RDL 3/2011, que las Comunidades Autónomas podrán, asimismo, atribuir la competencia para la resolución de los recursos al Tribunal Administrativo Central de Recursos Contractuales?. Por tanto, de la lectura conjunta, se puede concluir que la creación de un nuevo órgano, o varios, por parte de cada Comunidad Autónoma, no es obligatoria sino que éstas pueden optar por atribuir tales funciones al órgano estatal. En Cataluña, en concreto, se ha optado por crear un nuevo órgano.

El medio utilizado para su creación ha sido una ley de acompañamiento de la de presupuestos $^{8}$, la Ley autonómica $7 / 2011$, del 27 de julio, de medidas fiscales y finan-

6 Obligación que derivaría del propio tenor literal del art. 41.3 RDL 3/2011: «debiendo crear un órgano independiente».

7 Se requiere, en ese caso, de la realización de un convenio con la Administración General del Estado, donde se debe acordar cómo sufragar el gasto que se genere a causa de dicha atribución.

8 En esto, Cataluña no ha sido la única ni la pionera: v. la Ley 19/2010 de Castilla y León, de 22 de diciembre, de Medidas Financieras y de Creación del Ente Público Agencia de Innovación y financiación Empresarial y la Ley madrileña 9/2010, de 23 de diciembre, de Medidas Fiscales, Administrativas y Racionalización del Sector Público.

Más allá de las leyes de acompañamiento, ha habido otros sistemas para establecer la regulación del órgano encargado de la resolución de los recursos especiales en materia de contratación pública. Algunas Comunidades Autónomas han optado por una ley sectorial en materia contractual (por ejemplo, la ley aragonesa 3/2011, de 24 de febrero, de medidas en materia de Contratos del Sector Público de Aragón), o bien se ha optado directamente por incluir la creación del órgano en una disposición adicional de la ley de presupuestos (el País Vasco creó el Órgano Administrativo de Recursos Contractuales de la Comunidad Autónoma de Euskadi en la DA Octava de la Ley 5/2010, de 23 de diciembre, por la que se aprueban los 
cieras, que regula este órgano en su Disposición Final Cuarta, con un total de 17 largos apartados?.

A pesar de la temprana previsión de crear en Cataluña un órgano independiente para la resolución de los recursos contractuales ${ }^{10}$, antes de la aprobación de la ley catalana 7/2011, en Cataluña se aplicaba el régimen supletorio para las Comunidades Autónomas que se regulaba en la DT $2^{\text {a }}$ de la Ley $34 / 2010$ y que se encargaba de establecer un régimen transitorio hasta que las Comunidades Autónomas regularan ante quién incoar la cuestión de nulidad y ante quién interponer el recurso especial en materia de contratación. Este régimen transitorio, hoy mantenido en la DT $7^{\mathrm{a}} \mathrm{RDL}$ $3 / 2011$, establece que, en tanto no se determine el nuevo órgano independiente, son recurribles los actos mencionados en el artículo 40.2 RDL 3/2011, que se tramitarán de conformidad con lo establecido en los artículos 42 a 48 RDL 3/2011. La competencia para la resolución de los recursos sigue recayendo sobre los mismos órganos que la tuvieran atribuida con anterioridad, es decir, aquéllos regulados en el art. 37.4 de la Ley 30/2007 en la redacción originaria, previa a las modificaciones introducidas por la Ley 34/2010"1. No obstante la literalidad de la aplicación de este régimen transitorio ("[e]n tanto una Comunidad Autónoma no regule ante quién incoarse la cuestión de nulidad [... o o interponerse el recurso"), entendemos que debe extenderse su aplica-

Presupuestos Generales de la Comunidad Autónoma de Euskadi para el ejercicio 2011, disposición que guarda muy estrechas similitudes con la DA $4{ }^{\text {a }}$ de la ley catalana 7/2011).

9 Es conocida la controversia que provoca el uso de estas leyes de acompañamiento (cfr. García- Escudero, P., Técnica legislativa y seguridad jurídica: ¿hacia el control constitucional de la calidad de las leyes?, Civitas, 2010, pp. 152 y ss), si bien el TC español no ha declarado este tipo de leyes inconstitucionales hasta el momento, como muestra su reciente Sentencia 136/2011, de 13 de septiembre de 2011 (BOE núm. 245, de 11 de octubre de 2011), consecuencia del Recurso de inconstitucionalidad 1390-1999, interpuesto por 89 Diputados del Grupo Parlamentario Socialista del Congreso de los Diputados en relación con diversos preceptos de la Ley 50/1998, de 30 de diciembre, de medidas fiscales, administrativas y del orden social. Esta sentencia desestima el recurso de inconstitucionalidad interpuesto contra una ley de acompañamiento, y, en sus fundamentos jurídicos, afirma como totalmente constitucional el carácter heterogéneo de este tipo de leyes, así como su perfecto encaje en el sistema de fuentes; y también señala que este tipo de leyes no comparte las mismas limitaciones establecidas para las leyes presupuestarias. Por lo que respecta a las enmiendas introducidas en este tipo de leyes, se recuerda que deben guardar una conexión de homogeneidad mínima con la materia de la iniciativa.

10 Téngase en cuenta la Ley catalana 26/2009, de 23 de diciembre, de medidas fiscales, financieras y administrativas, en cuya DA Cuarta se autoriza al Gobierno para crear "un órgano colegiado independiente para el conocimiento y la resolución de los recursos especiales en materia de contratación que se interpongan con relación a los procedimientos de contratación de la Administración de la Generalidad, y también de los entes, las entidades y los organismos que integran su sector público, con el fin de garantizar la aplicación correcta de la Directiva 89/665/CEE del Consejo [... ] y la Directiva 2007/66/CE del Parlamento Europeo y del Consejo". Este órgano debía crearse durante 2010, y así se intentó, como atestigua un proyecto de decreto de creación del Tribunal de Contratos del Sector Público fechado en el año 2010, que proponía un órgano colegiado e independiente funcionalmente, compuesto por siete miembros y adscrito al Departamento de Economía y Finanzas de la Generalidad de Cataluña (elperfildelcontratante.com/pdf.php?cod=214\&pdf=2), (última consulta: 27 de septiembre de 2011).

11 Por tanto, se trata de un órgano no independiente, lo cual supuso temporalmente un incumplimiento de la Directiva 2007/66/CE. 
ción no hasta que los extremos requeridos hayan sido regulados, sino hasta que el órgano independiente competente inicie sus actividades ${ }^{12}$.

\section{III.2. Naturaleza jurídica}

El Órgano de Recursos Contractuales de Cataluña se configura como un órgano administrativo especializado de carácter unipersonal que actúa con plena independencia funcional y adscrito a un departamento de la Administración de la Generalidad de Cataluña.

Su carácter de órgano especializado está establecido en el apartado 1 de la DA $4^{\text {a }}$. La materia de especialización es la contratación pública, como así ponen de manifiesto las distintas disposiciones que le dan contenido, como son la regulación de la competencia material del propio órgano (DA $4^{\mathrm{a}}$.4) y algunas disposiciones relativas a su titular: por ejemplo, se establece, dentro de los requisitos para ser titular del órgano, la preferencia por los profesionales directamente relacionados con la contratación pública (DA $4^{\mathrm{a}}$.10.a) y una competencia profesional acreditada en las materias propias del órgano (DA $4^{\mathrm{a}}$.10.c); además, durante el proceso de selección, se obliga a los candidatos a hacer constar en su currículum una serie de datos profesionales "especialmente para acreditar de forma fehaciente la experiencia y la dedicación en el ámbito del derecho público relacionado con la contratación pública" (DA 4 a .11 in fine).

Este Órgano se crea con carácter unipersonal, tal y como establece la DA $4^{\mathrm{a}}$.9. No obstante, una vez puesto en funcionamiento, la ley contempla la posibilidad de su transformación en un órgano colegiado (DA $4^{\mathrm{a}}$.17). Los requisitos habilitantes de tal transformación son:

- Que el volumen y la especificidad de los asuntos que son competencia del Órgano Administrativo de Recursos Contractuales de Cataluña aconsejen la modificación del carácter unipersonal; y

- Que el órgano competente en materia de evaluación y supervisión de la contratación pública'³ efectúe una propuesta "fundamentada en la evolución

12 Cuestión que ha sido relevante en Cataluña porque, a diferencia de otras regulaciones autonómicas, la catalana carece de normativa transitoria aplicable ante la creación de este nuevo órgano. Compárese, por ejemplo, con la Ley aragonesa 3/2011, de 24 de febrero, de medidas en materia de contratos del Sector Público de Aragón, en cuyas disposiciones transitorias se regula el tratamiento de los expedientes de contratación ya iniciados en el momento de la entrada en vigor de dicha ley y, en tanto se constituya efectivamente el Tribunal Administrativo de Contratos Públicos de Aragón, atribuye sus funciones a los vocales de un órgano ya existente y en funcionamiento (la Junta Consultiva de Contratación Administrativa de la Comunidad Autónoma de Aragón).

Afortunadamente, esta cuestión ya no va a ser problemática en cataluña: el día 1 de junio de 2012 entró en funcionamiento el Órgano Administrativo de Recursos Contractuales de Cataluña.

13 Actualmente, se trata de la Oficina de Supervisión y Evaluación de la Contratación Pública, la cual se estudia más adelante (vid nota al pie número 19). 
creciente que haya podido experimentar el número total de recursos presentados" que aconseje la modificación del carácter unipersonal; tras lo cual será necesario

— Que un decreto del Gobierno autonómico opere la transformación ${ }^{14}$.

Si se operara en el futuro la transformación en órgano colegiado, la denominación de este Órgano cambiaría a la de Tribunal Catalán de Contratos del Sector Público ${ }^{15}$.

La plena independencia funcional de este Órgano en el ejercicio de sus competencias está declarada explícitamente en dos apartados de la DA $4^{\mathrm{a}}$, el $1^{\circ}$ y el $5^{\circ}$, y en otros se determinan varios aspectos de la misma, como la ausencia de vinculación jerárquica del Órgano (DA $4^{\mathrm{a}} \cdot 7$ ), la independencia personal y la autonomía material (DA $4^{\mathrm{a}}$, aps. 13 y 14$)^{16}$. Como es conocido, ésta es una de las principales novedades introducidas por la modificación de la legislación básica de contratos operada por la Ley 34/2010, de 5 de agosto, ${ }^{17}$ pues debe recordarse que en la redacción originaria de la Ley 30/2007, el art. 37.4 establecía como competentes para la resolución del recurso administrativo especial en materia de contratación al "órgano de contratación, cuando se trate de contratos de una Administración Pública, o el titular del departamento, órgano, ente u organismo al que esté adscrita la entidad contratante o al que corresponda su tutela, si ésta no tiene el carácter de Administración Pública”18.

El vigente art. 41.1 RDL 3/2011 crea el Tribunal Administrativo Central de Recursos Administrativos en el ámbito de la Administración General del Estado como un órgano independiente; y, para el ámbito autonómico, se establece en el art. 41.2 RDL 3/2011 que "la competencia para resolver los recursos será establecida por sus normas respectivas [autonómicas], debiendo crear un órgano independiente”. Debe tenerse presente que, de acuerdo con la DF $2^{\mathrm{a}} \mathrm{RDL}$ 3/2011, el art. 41.2 tiene carácter básico, con lo que puede concluirse que la necesidad de que el órgano autonómico sea independiente procede de la Directiva 2007/66/CE y se garantiza para todo el ámbito estatal en la legislación básica. Este aspecto, es además crucial como elemento garantizador del derecho a una buena administración previsto en el art. 30 del Estatuto de Autonomía de Cataluña.

14 Este decreto deberá incluir el reglamento de organización y funcionamiento del Tribunal (v. DA $\left.4^{\mathrm{a}} \cdot 17 \cdot \mathrm{e}\right)$.

15 Los demás aspectos de este Tribunal serán tratados en el apartado correspondiente a la estructura del Órgano (III.4).

16 Las garantías de la independencia funcional del Órgano se tratan con mayor detalle en el apartado III.4.3 de este estudio.

17 En cumplimiento de las exigencias de la Directiva 2007/66/CE.

18 Entonces, la consideración que se hacía para la regulación autonómica era: "Sin perjuicio de lo que, para el ámbito de las Comunidades Autónomas, establezcan sus normas respectivas que, en todo caso, deberán atribuir esta competencia a un órgano cuyas resoluciones agoten la vía administrativa [... ]". 
El Órgano de Recursos Contractuales de Cataluña se encuentra adscrito a un departamento de la Generalidad de Cataluña. De acuerdo con la DA $4^{\mathrm{a}} \cdot 5$, el Órgano se adscribe al mismo departamento "al que esté adscrito el órgano competente en materia de evaluación y supervisión de la contratación pública”. El órgano así referido es, actualmente, la Oficina de Supervisión y Evaluación de la Contratación Pública ${ }^{19}$, regulada por el Decreto 325/2011, de 26 de abril, de reestructuración del Departamento de la Presidencia.

Ese decreto establece en su art. 3.2.c que la Oficina de Supervisión y Evaluación de la Contratación Pública depende de la Secretaría del Gobierno, y, de acuerdo con el art. 1.1.a, la Secretaría del Gobierno es una de las unidades directivas del Departamento de la Presidencia. Por tanto, como la Oficina está adscrita al Departamento de la Presidencia, hay que entender, en virtud de la DA $4^{a} \cdot 5$ Ley $7 / 2011$, que el Órgano de Recursos Contractuales de Cataluña se encuentra también adscrito a ese Departamento.

La adscripción determinada anteriormente también es importante a efectos de conocer la ubicación de la sede del Órgano Administrativo de Recursos Contractuales de Cataluña porque la ley determina que tiene la sede en el mismo lugar donde se encuentren los órganos centrales del departamento de adscripción. Esta ubicación determinada por la ley puede ser modificada posteriormente por el Gobierno en caso de que su traslado se considere necesario para su correcto funcionamiento (DA $\left.4^{\mathrm{a}} \cdot 6\right)$.

19 La Oficina de Supervisión y Evaluación de la Contratación Pública fue creada por el Decreto 203/2011, de 18 de enero, el cual fue derogado expresamente por el Decreto 325/2011, de 26 de abril. De acuerdo con la normativa vigente, esta Oficina, que tiene rango orgánico de dirección general, tiene asignadas distintas funciones en materia de contratación pública: velar por la aplicación del principio de transparencia administrativa en la contratación pública en la Administración de la Generalidad y en su sector público, y proponer todas las medidas tendentes a su mejora y efectividad; establecer directrices y unificar criterios de actuación en materia de contratación pública de la Administración de la Generalidad y de su sector público; hacer el seguimiento de la puesta en marcha y ejecución de las disposiciones aprobadas y de los acuerdos adoptados en materia de contratación pública por los órganos competentes y evaluar su aplicación; proponer los cambios a introducir en el ámbito de la contratación pública para la mejora de los aspectos administrativos, técnicos y económicos que considere apropiados; dirigir las actuaciones relacionadas con la consecución de los objetivos establecidos en la normativa vigente sobre contratación pública, sin perjuicio de las funciones que correspondan a otros órganos; asesorar sobre los contenidos de los proyectos de disposiciones de carácter general en materia de contratación pública; analizar, supervisar y controlar los procedimientos, procesos y sistemas de gestión y organización de la contratación pública de la Administración de la Generalidad y de su sector público, sin perjuicio de las funciones que correspondan a la Intervención General de la Generalidad de Cataluña; dirigir y coordinar los medios electrónicos en la contratación pública, sin perjuicio de las funciones de la Secretaría Técnica de la Junta Consultiva de Contratación Administrativa de la Generalidad de Cataluña; velar por el cumplimiento de la normativa vigente en materia de contratación pública y garantizar la introducción de mejoras en los procedimientos, procesos y sistemas de gestión y organización de la contratación pública de la Administración de la Generalidad y de su sector público; dar apoyo a la Secretaría del Gobierno en el ejercicio de su función de supervisar y coordinar las actuaciones en materia de contratación pública, y facilitar el ejercicio de esta función (v. art. 6.1 Decreto $200325 / 2011$, de 26 de abril). 


\section{III.3. Ámbito competencial}

Al abordar el ámbito competencial, es preciso detenerse a examinar tanto la competencia material como la extensión territorial de ésta.

\section{III.3.1. Territorial}

El apartado 2 de la DA $4^{\text {a }}$ es el encargado de establecer la extensión competencial del Órgano. Según éste, "El Órgano Administrativo de Recursos Contractuales de Cataluña ejerce sus funciones en el ámbito de la Administración de la Generalidad, de las entidades y los organismos que forman parte de su sector público que tienen la consideración de poderes adjudicadores, de las administraciones locales integradas en su territorio, así como de las entidades y organismos de la Administración local que tienen la consideración de poderes adjudicadores."

De acuerdo con el párrafo citado, en principio todos los municipios de Cataluña quedarían sometidos al Órgano autonómico. No obstante, se regula a continuación, en la misma DA $4^{\mathrm{a}} .2$, la posibilidad, para municipios de más de 50.000 habitantes, de crear su propio órgano con competencia para resolver los recursos de su ámbito local (y su sector público) ${ }^{20}$.

Esta regulación del ámbito local por parte de la Comunidad Autónoma es lo previsto por la legislación básica: el art. 41.4 RDL 3/2011, primer párrafo, dispone que la competencia para resolver los recursos en el ámbito de las Corporaciones Locales quedará establecida por la Comunidad Autónoma a la que pertenezcan siempre que dicha Comunidad tenga atribuidas facultades normativas y ejecutivas en materia de régimen local y de contratación; las cuales tiene atribuidas Cataluña ${ }^{21}$.

A pesar de que en Cataluña está regulada la cuestión local, debe tenerse presente que, de no haberse regulado, sería aplicable, supletoriamente en caso de laguna, el art. 41.4 RDL 3/2011, segundo párrafo, el cual establece que, en caso de ausencia de regulación autonómica sobre los recursos en el ámbito de las Corporaciones Locales, la competencia recaerá sobre el mismo órgano al que la Comunidad Autónoma en que se integren haya atribuido la competencia para la resolución de los recursos de su propio ámbito.

\section{III.3.2. Material}

La competencia material se encuentra regulada en la DA $4^{\mathrm{a}} \cdot 4$. En concreto, el Órgano tiene competencia material para resolver los recursos especiales en materia

20 Técnica previamente prevista en la regulación del órgano del País Vasco, que ha dado pie a la creación de órganos forales de recursos.

21 Arts. 159 y 160 del Estauto Autonomía de Cataluña. 
de contratación interpuestos contra los actos referidos en el art. 40.2 RDL 3/2011 (en conexión a los demás apartados del mismo art. 40) y las decisiones referidas a la adopción de las medidas provisionales que, con anterioridad a la interposición del recurso especial, soliciten las personas legitimadas; también es competente para resolver las cuestiones de nulidad contractual establecidas en los supuestos especiales del art. 37 RDL 3/2011; y, finalmente es competente para resolver los mismos extremos anteriormente nombrados pero en relación a los sectores del agua, la energía, los transportes y los servicios postales (las remisiones normativas en este caso se hacen a los arts. 101, 103 y 109-111 de la Ley 31/2007, de 20 de octubre, sobre procedimientos de contratación en los sectores del agua, la energía, los transportes y los servicios postales).

\section{III.4. Estructura}

\section{III.4.1. Titular}

Como ya se ha dicho, el Órgano autonómico de Cataluña se crea inicialmente como un órgano unipersonal (DA $4^{\mathrm{a}} \cdot 9$ ). Los requisitos para ser designado titular de este Órgano se encuentran en la DA $4^{\mathrm{a}} .10$, y son: ser funcionario de carrera de los cuerpos o escalas A1 (con experiencia laboral superior a 15 años, preferentemente en el ámbito del derecho administrativo relacionado con la contratación pública), disponer de la licenciatura en derecho y de una competencia profesional acreditada en las materias propias del Órgano. Estos requisitos son igualmente necesarios para el sustituto (v. DA $\left.4^{\mathrm{a}} \cdot 12 . \mathrm{III}\right)$.

Su proceso de selección se rige por los principios de igualdad, publicidad, mérito y capacidad, y se inicia mediante convocatoria pública, en que pueden participar todos aquellos funcionarios de carrera que cumplan con los requisitos referidos en el párrafo anterior. Para participar, junto con la solicitud es necesario incluir un currículum en que conste: la titulación académica, la pertenencia a los cuerpos o escalas exigidos, los años de servicio prestados, los puestos de trabajo desarrollados en las administraciones públicas y demás méritos que se consideren adecuados para acreditar la experiencia y la dedicación en el ámbito de la contratación pública.

En caso de transformación del Órgano en Tribunal Catalán de Contratos del Sector Público, tenemos que acudir a la DA $4^{\mathrm{a}}$.17 para conocer las especialidades referidas al titular. Realizada la transformación, la persona designada como titular del Órgano pasaría a ocupar el cargo de Presidente del Tribunal. Además del Presidente, el Tribunal también estaría compuesto por dos vocales, número que podría ser aumentado por reglamento, pero no reducido, por causas debidamente acreditadas referidas al volumen de asuntos que le sean sometidos.

Tanto el Presidente como los vocales comparten la misma regulación de requisi202 tos y procedimiento para ser designados miembros del Tribunal que la expuesta para 
el órgano unipersonal ${ }^{22}$, con la única especialidad de que, en el proceso de selección de los vocales, al menos uno, debe ser designado a propuesta conjunta de la Asociación Catalana de Municipios y la Federación de Municipios de Cataluña.

\section{III.4.2. Estatuto personal}

El estatuto personal del titular del Órgano está regulado en la DA $4^{\mathrm{a}} .12^{23}$, y se refiere a varios aspectos.

La designación del titular del Órgano la hace el titular del Departamento de la Presidencia mediante resolución que, además del nombramiento ${ }^{24}$, debe establecer las retribuciones ${ }^{25}$, el régimen jurídico del Órgano y las condiciones para la sustitución del titular en los supuestos de vacante, ausencia o enfermedad. Esta resolución será publicada en el Diario Oficial de la Generalidad de Cataluña.

El mandato es de cinco años a contar desde la toma de posesión ${ }^{26}$, que debe llevarse a cabo una vez publicado el nombramiento expuesto anteriormente. El titular es reelegible por períodos sucesivos de cinco años sin necesidad de realizarse nuevos procesos de selección ${ }^{27}$. Durante su mandato, el titular es inamovible, está sujeto al mismo régimen de incompatibilidades que corresponde a los altos cargos de la Generalitat ${ }^{28}$ y ejerce sus funciones con la denominación de director o directora del Órgano Administrativo de Recursos Contractuales de cataluña ${ }^{29}$.

22 Como se ha dicho en el párrafo anterior, se exceptúa al primer titular del Órgano durante cuyo ejercicio se opere la transformación, ya que pasaría directamente a Presidente del Tribunal sin tener que presentarse otra vez a un proceso selectivo, siempre y cuando no haya expirado su mandato en el Órgano.

23 Este apartado 12 ha sido objeto de modificación por el art. 79 de la Ley catalana 5/2012, de 20 de marzo, de medidas fiscales, financieras y administrativas y de creación del impuesto sobre las estancias en establecimientos turísticos.

24 El nombramiento da lugar a la declaración de la situación administrativa de servicios especiales (situación administrativa de los funcionarios de carrera regulada, a nivel básico, en el art. 87 de la Ley 7/2007, de 12 de abril, del Estatuto básico del empleado público, y, a nivel autonómico catalán, en el art. 88 del Decreto Legislativo 1/1997, de 31 de octubre, por el que se aprueba la refundición en un Texto único de los preceptos de determinados textos legales vigentes en Cataluña en materia de función pública.

25 Sus retribuciones deben asimilarse a las correspondientes al personal funcionario de la Administración de la Generalitat (v. DA 4. ${ }^{a}$.12.I.c.). En concreto, la primera resolución de nombramiento fija unas retribuciones correspondientes al nivel 30.2 de las establecidas para los funcionarios de la Administración de la Generalitat (v. Resolución PRE/1014/2012, de 31 de mayo, por la cual se resuelve la convocatoria para la provisión, por el sistema de libre designación, de un puesto de trabajo adscrito al Departamento de la Presidencia, en DOGC núm. 6139, de 31 de mayo de 2012, pp. 28123-28124).

26 De acuerdo con la DA 4 a .15 , el Órgano inicia su actividad el mismo día de la publicación del nombramiento del titular y una vez ha tomado posesión del cargo.

27 A título de curiosidad, apréciese la diferencia con el Tribunal Administrativo Central de Recursos Contractuales, la duración del nombramiento de cuyos miembros es de seis años no prorrogables.

28 Regulado en la Ley catalana 13/2005, de 27 de diciembre, del régimen de incompatibilidades de los altos cargos al servicio de la Generalitat.

29 El primer director del Órgano fue designado como tal en la ya citada Resolución PRE/1014/2012, de 31 de mayo. 
Respecto al Tribunal, el estatuto personal de los miembros es idéntico al del titular del Órgano, de acuerdo con la DA $4^{\mathrm{a}}$. 17.d.

\section{III.4.3. Garantías de independencia funcional}

Las garantías de la independencia funcional del órgano quedan plasmadas en distintos preceptos de la DA $4^{\mathrm{a}}$.

En primer lugar, el nombramiento de los miembros (sea del Órgano o del Tribunal) está sometido a una serie de requisitos previos que pretenden cualificar a los solicitantes. Además, el currículum a aportar junto con la solicitud de admisión en el proceso de selección, se centra exclusivamente en aquellos datos relevantes para determinar la capacidad profesional del solicitante. Finalmente, conviene recordar que la provisión del puesto de titular debe ajustarse a los principios de igualdad, publicidad y mérito (DA $\left.4^{\mathrm{a}} \cdot 11\right)$.

En segundo lugar, el mandato de cinco años se ejerce con inamovilidad y sólo es posible la remoción con causa tasada y determinante o grave. En la DA $4^{\mathrm{a}} .13$ se tasan las causas de cese o remoción del titular, que son: la defunción, la finalización del mandato, la renuncia formalizada por escrito y aceptada por el titular del Departamento de Presidencia, la pérdida de la condición de funcionario, el incumplimiento grave de sus obligaciones, la condena por sentencia firme a pena privativa de libertad o de inhabilitación absoluta o especial para ocupaciones o cargos públicos, la incapacidad sobrevenida para el ejercicio del cargo y la pérdida de la nacionalidad. La remoción derivada de las cinco últimas causas debe ser acordada por el titular del Departamento de la Presidencia previa instrucción con audiencia del interesado. Tanto el cese como la remoción se producen cuando se da la circunstancia o tiene efectos el acto que la determina. En todo caso, deben publicarse en el Diario Oficial de la Generalidad de Cataluña.

En tercer lugar, se pretende garantizar la independencia mediante la puesta a disposición del Órgano de los medios personales y materiales necesarios para el ejercicio de sus funciones de manera adecuada, tal como establece la DA $4^{\mathrm{a}} \cdot 14$. La Administración de la Generalidad es la encargada de garantizar la disponibilidad de tales medios.

\section{III.4.4. El procedimiento}

En el apartado 8 de la DA $4^{\text {a }}$ se establece la regulación del procedimiento de interposición, tramitación y resolución de los recursos y demás actos competencia del Órgano.

En primer lugar hace una referencia genérica según la cual se aplicaría "el proce-

204 dimiento establecido por la normativa sobre contratación del sector público", si bien 
a continuación se concreta la afirmación en una serie de preceptos. Para los recursos especiales, se citan los arts. 310-319 de la ley 30/2007 (hoy, arts. 40-49 RDL 3/2011) y de la Ley 31/2007, los arts. 101-108.

Para la cuestión de nulidad, hay que añadir las especialidades de los arts. 39 Ley 30/2007 (39 también en el RDL 3/2011) y 111 Ley 31/2007. Finalmente, se citan las reglas de aplicación supletoria, que son las reglas generales de régimen jurídico y procedimiento: la Ley estatal 30/1992, de 26 de noviembre, y la Ley catalana 26/2010, de 3 de agosto, de régimen jurídico y de procedimiento de las administraciones públicas de Cataluña.

Hasta aquí, pues, la exposición de lo regulado expresamente en la normativa de creación del Órgano Administrativo de Recursos Contractuales de Cataluña, si bien hay que tener en cuenta queo muchas de las cuestiones que por el momento se perfilan imprecisas o incluso directamente no reguladas, esperan de la previsible y próxima regulación reglamentaria, a la que se refieren dos apartados de la DA $4^{\text {a }}$ : el 16 (donde se autoriza al Gobierno para establecer las disposiciones necesarias para el funcionamiento correcto del Órgano) y el 17.e (donde se establece la obligación de incorporar el reglamento de organización y funcionamiento del Tribunal en el decreto de transformación del Órgano).

\section{ALGUNAS REFLEXIONES FINALES: CONTRATACIÓN Y DERECHO A UNA BUENA ADMINISTRACIÓN}

Para concluir queremos señalar cómo la configuración de órganos administrativos independientes que se deriva de las directivas comunitarias y de la legislación española y autonómica supone un avance en el reforzamiento y la protección del derecho a una buena administración en la gestión administrativa contractual, como pone de relieve por ejemplo Gimeno Feliu ${ }^{30}$. Efectivamente, la garantía de la resolución de los recursos especiales y cuestiones de nulidad por órganos que actúen de forma objetiva e imparcial refuerza la protección de los derechos e intereses de los operadores económicos en el mercado y, simultáneamente, robustece la buena administración en este ámbito, al fortalecer el trato de sus asuntos "imparcial y equitativamente" (art. 41 de la Carta de Derechos Fundamentales de la Unión Europea, consagrando el derecho a una buena administración) y garantizar que, en el caso específico catalán, todas las personas van a tener el derecho "a ser tratadas por los poderes públicos de Cataluña, en los asuntos que les afectan, de forma imparcial y objetiva" (art. 30 del vigente Estatuto de Autonomía de Cataluña, reconociendo el derecho a una buena administración) ${ }^{31}$.

30 Gimeno Feliu, J.M., Las reformas legales de la ley 30/2007, de Contratos del Sector Público. Alcance y Efectos Prácticos, Civitas, 2011, pp. 140 y ss. especialmente.

31 Ponce Solé, J., “Derecho Público y buena administración”, Jurisprudencia Argentina, 2011-III, 14 de septiembre de 2011, pp. 3 y ss., consultable en:

http://portalanterior.abeledoperrot.com/Noticias/MostrarNoticiaNew.asp?cod=8601\&tipo=2 
Efectivamente, reforzar legalmente los principios constitucionales de objetividad e imparcialidad de la actuación administrativa (art. 103.1 CE) mediante el diseño organizativo administrativo, en este caso, es un modo de reforzar las obligaciones de buena administración y, por tanto, de contribuir a que las decisiones administrativas en vía de recurso en materia de recursos especiales y acciones de nulidad promuevan el resto de principios constitucionales de buena administración (interdicción de la arbitrariedad, art. 9.3 CE, eficiencia y economía en el manejo de caudales públicos, art. 31.2 (E) y tutelen adecuadamente, en definitiva, el derecho a una buena administración de los afectados. Y garantizar la buena administración es un modo de evitar la mala administración y, en el más extremo de los supuestos, la corrupción en el ámbito de la contratación ${ }^{32}$. 